\title{
KRAS-related long noncoding RNAs in human cancers
}

\author{
Mahsa Saliani ${ }^{1,2}$, Amin Mirzaiebadizi $^{1}$, Ali Javadmanesh ${ }^{3,4}$, Akram Siavoshi $^{5}$ and Mohammad Reza Ahmadian $\left(\mathbb{D}^{1 凶}\right.$ \\ (c) The Author(s) 2021
}

\begin{abstract}
KRAS is one of the most widely prevalent proto-oncogenes in human cancers. The constitutively active KRAS oncoprotein contributes to both tumor onset and cancer development by promoting cell proliferation and anchorage-independent growth in a MAPK pathway-dependent manner. The expression of microRNAs (miRNAs) and the KRAS oncogene are known to be dysregulated in various cancers, while long noncoding RNAs (IncRNAs) can act as regulators of the miRNAs targeting KRAS oncogene in different cancers and have gradually become a focus of research in recent years. In this review article, we summarize recent advances in the research on IncRNAs that have sponging effects on KRAS-targeting miRNAs as crucial mediators of KRAS expression in different cell types and organs. A deeper understanding of IncRNA function in KRAS-driven cancers is of major fundamental importance and will provide a valuable clinical tool for the diagnosis, prognosis, and eventual treatment of cancers.
\end{abstract}

Cancer Gene Therapy (2022) 29:418-427; https://doi.org/10.1038/s41417-021-00381-x

\section{INTRODUCTION}

KRAS is a small GDP/GTP-binding protein that transduces extracellular signals and induces intracellular responses. KRAS cycles between an inactive, GDP-bound ("off") state, and an active, GTP-bound ("on") state. This off/on cycle is based on GDP/GTP exchange and GTP hydrolysis reactions stimulated by RAS-specific guanine nucleotide exchange factors (GEFs) and GTPaseactivating proteins (GAPs), respectively [1]. GTP-bound KRAS transduces signals to its downstream effectors and thus activates multiple signaling pathways $[2,3]$. Therefore, activated KRAS controls various cellular processes, including survival, growth, proliferation, differentiation, and apoptosis, all of which are known as hallmarks of cancer [4]. Somatic mutations in KRAS trigger the robust gain-of-function effects of oncogenic KRAS and neoplastic signal transduction owing to the reduction in GTP hydrolysis and resistance to GAP function [5, 6].

The KRAS oncogene has been extensively studied in human tumor malignancies $[7,8]$. Intensive efforts to understand the mechanisms underlying the intracellular trafficking, regulation, and signaling pathways of KRAS have suggested several therapeutic strategies [9]. Despite its well-recognized importance in cancer promotion, only a few efforts in the past four decades have resulted in approved clinical therapeutic strategies for KRAS-mutant cancers [9-11]. Additionally, KRAS mutation is an important predictive marker in determining resistance to EGFRtargeted therapies [12]. Thus, further studies are needed to elucidate the mechanisms responsible for the modulation of KRAS to evaluate other potential therapeutic approaches.

Long noncoding RNAs (IncRNAs) are a class of noncoding RNAs (ncRNAs) with a minimum length of 200 nucleotides, which have been well studied in the context of RNA-based therapeutics $[13,14]$. Although only a small fraction of known IncRNAs have been functionally characterized, there is growing evidence of their involvement in a variety of biological processes, human diseases, and malignancies [15]. These molecules, as the key regulators of gene expression, play essential roles in a wide variety of biological processes and signaling pathways involved in the progression of many human cancers [16-19]. Emerging evidence has suggested that various IncRNAs are likely to function as competing endogenous RNAs (ceRNAs). These IncRNAs act as oncogenes by sponging tumor suppressor microRNAs (miRNAs) [20, 21], thereby indirectly regulating the expression of the genes targeted by these miRNAs [22] (Fig. 1). Considering the wide diversity of miRNAs and their high capacity for regulating hundreds of genes, many driver oncogenes, such as ERBB2, BRAF, EGFR, MYC, SRC, and BCL2, are targeted by miRNAs [23-25]. In this regard, many tumor suppressor miRNAs have inhibitory effects on KRAS-associated tumrigenesis by downregulating KRAS expression [26, 27]. Therefore, oncogenic IncRNAs, as sponges of tumor suppressor miRNAs that target KRAS, promote cancer development via the upregulation of the KRAS oncogene [28-30].

It is evident that ceRNAs and miRNA response elements (MREs) are two essential components of the 'sponge effect' [31]. MREs are seed regions of 2-8 nucleotides in the $5^{\prime}$ region of miRNA [32]. The ability of a miRNA to bind to its mRNA target and IncRNA via its MRE provides competition between mRNA and IncRNA for interaction with their target miRNA. The binding of IncRNA to miRNA as a ceRNA prevents the latter from recognizing mRNA and consequently results in its silencing. This interaction leads to the regulation of MREs on the targets, which plays an important role in posttranscriptional regulation and is known as the sponging effect [31] (Fig. 1).

Identification of mechanisms involved in KRAS regulation by IncRNAs is expected to greatly enhance our understanding of the

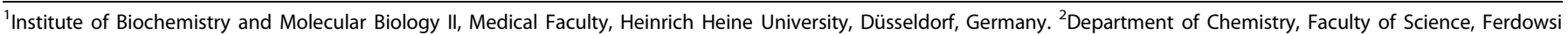

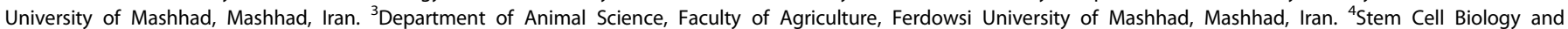

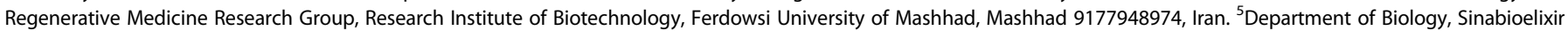
Group, Alborz Health Technology Development Center, Tehran, Iran. ${ }^{\bowtie}$ email: reza.ahmadian@hhu.de

Received: 17 March 2021 Revised: 29 June 2021 Accepted: 11 August 2021

Published online: 6 September 2021 


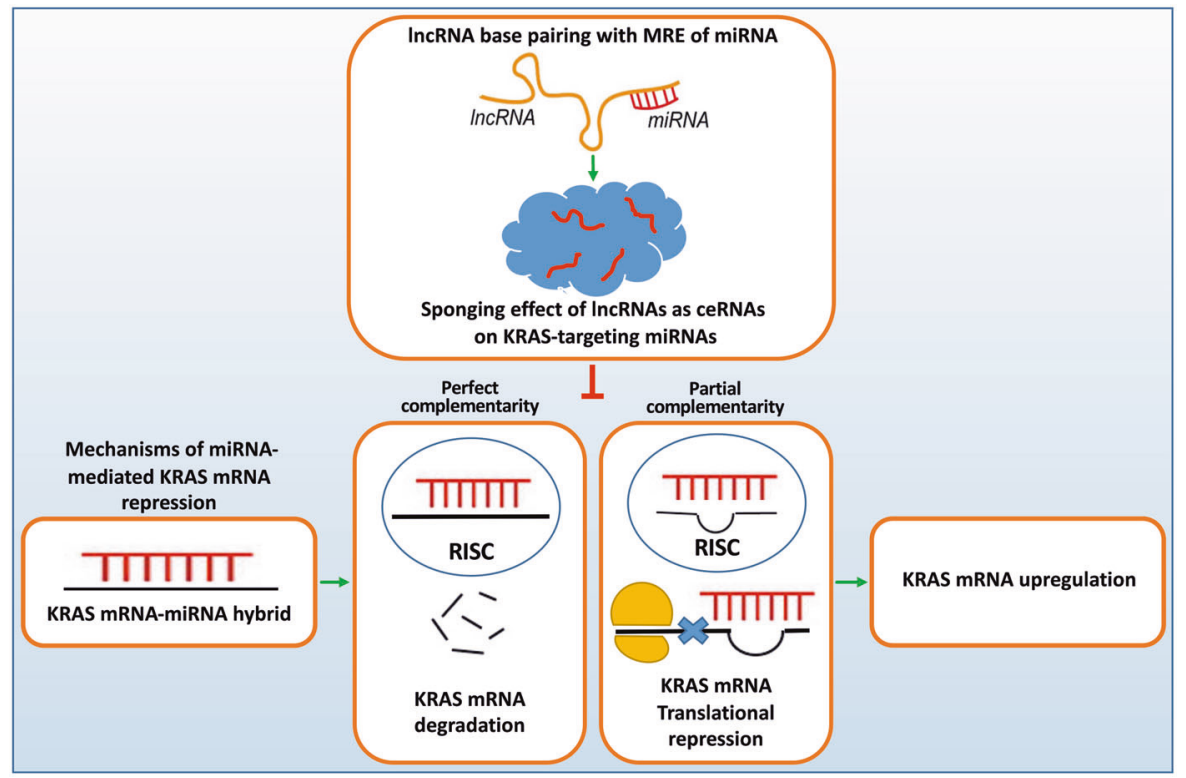

Fig. 1 Mechanism of KRAS gene regulation by oncogenic IncRNAs through sponging effects. As key gene regulators, tumor suppressor miRNAs bind to their targets and interfere with translation. The RNA-induced silencing complex (RISK) guides the antisense strand of the miRNA to bind to its target KRAS mRNA sequence in a complementary manner, forming a double-stranded helix. Perfect complementarity results in endonucleolytic cleavage, while partial complementarity subjects mRNA to translational repression. Oncogenic IncRNAs act as ceRNA decoys by presenting complementary sequences with MREs to sponge miRNAs from their target KRAS mRNAs. IncRNAs consequently promote KRAS mRNA stabilization and thus its upregulation.

mechanisms of tumorigenesis associated with KRAS regulation. While the sponging effect of IncRNAs on miRNAs that target KRAS seems to be one of the key mechanisms by which KRAS is regulated, details of other regulatory mechanisms remain to be elucidated. The association of IncRNAs with various regulatory apparatuses, such as chromatin remodeling factors, transcription factors, splicing machinery, nuclear trafficking modulators, and miRNAs, shows the complexity of their regulatory approaches $[33,34]$. Therefore, to understand other regulatory effects of IncRNAs on KRAS expression, the role of all interactions between IncRNAs and other macromolecules, such as DNA, RNA, and proteins, in the regulation of gene expression should be considered. Based on the different methods of gene regulation by IncRNAs, IncRNAs are divided into guides, scaffolds, signaling molecules, decoys, and miRNA sponges, which affect the pretranscription, transcription, and posttranscriptional levels of gene expression [34, 35]. It is now evident that silencing G4 elements in the core promoter region of oncogenes such as KRAS is a highly valuable and new molecular target in the treatment of cancer [36]. Some innovative approaches have suggested that IncRNAs containing G4 structures as molecular decoys for G4binding proteins prevent $\mathrm{G} 4$ formation in the promotor region of oncogenes, which leads to gene transcription [37]. Therefore, the determination of whether IncRNAs inhibit G4 element formation in the promotor region of KRAS reveals other mechanisms by which IncRNAs regulate KRAS expression at the pretranscription level. The results of another study demonstrated that KRASIM, a highly conserved microprotein encoded by the putative IncRNA NCBP2-AS2, plays a tumor-suppressive role by interacting with KRAS in HCC cells. KRASIM, as the first KRAS-binding protein encoded by a IncRNA, suppresses the protein level of KRAS and inhibits the ERK signaling pathway. Therefore, sequestration of the KRAS protein with peptides encoded by IncRNAs can be considered as an alternative IncRNA-associated posttranscriptional regulatory mechanism [38].

While IncRNAs have the capacity to regulate KRAS expression, abnormal levels of KRAS, one of the mediators of many cellular signaling pathways, reciprocally cause diverse molecular alterations, such as dysregulation of IncRNA expression. KRAS amplification has been shown to be a secondary means of KRAS activation, leading to its overexpression and neoplastic transformation. It was found that the levels of a KRAS-responsive IncRNA called KIMAT1 correlate with the KRAS levels and play a positive role in maintaining tumorigenesis [39]. Another study revealed that oncogenic RAS-induced IncRNA 1 (Orilnc1) can be regulated by the RAS-RAF-MEK-ERK pathway and is required for cell proliferation in RAS/BRAF-dependent human cancers [40].

The diversity of miRNAs with their various MREs provides a greater possibility for communication between different miRNAs and ceRNAs, two irreplaceable contributors to the sponging effect. This hypothesis suggests that the sponging effect is a key molecular mechanism underlying the networks corresponding to miRNAs, oncogenic IncRNAs, and many related oncogenic drivers that control various cancer-related biochemical processes. While $K R A S$-associated miRNAs have been widely studied in cancer, the role of KRAS-related IncRNAs in promoting cancer progression needs to be carefully examined. The ever-increasing number of $K R A S$-specific IncRNAs strongly indicates their potential contribution to and critical roles in the entire process of KRAS-driven carcinogenesis. This review compiles the current knowledge of KRAS-related oncogenic IncRNAs by considering their aberrant expression and their mechanism of action through sponging effects on KRAS-targeting miRNAs.

\section{NONCODING RNAS IN KRAS-DRIVEN CANCERS}

The noncoding transcriptome consists of a variety of different RNA types, such as transfer RNA (tRNAs), ribosomal RNAs (rRNAs), small nuclear RNAs (snRNAs), small nucleolar RNAs (snoRNAs), circular RNAs (circRNAs), miRNAs, and IncRNAs. Other than miRNAs and IncRNAs, as noncoding RNAs that play roles in tumorigenesis, accumulating evidence indicates that altered processing or activity of other RNA species can similarly contribute to cancer [13]. Intact tRNAs and tRNA fragments (tRFs) are correlated with tumorigenesis [41]. Upregulation of specific tRNA expression in breast cancers by the enhancement 
of the translation of specific transcripts has been demonstrated in the progression of metastasis [42]. In particular, a proportion of tRFs that are of the same size as miRNAs and associated with Argonaute are able to function as miRNAs. To confirm the oncogenic activity of tRFs, altered levels have been indicated in leukemia and solid cancers [42-44]. It has been reported that some tRNA fragments, such as ts-47s and ts-46s, are upregulated by KRAS and PIK3CA mutations, respectively, leading to breast cancer chemoresistance $[45,46]$. The results indicated that the expression of tRFs can be influenced by oncogenic mutations with a possible role in the promotion of carcinogenic processes. Other findings have demonstrated that the expression of different tRNAs corresponds to differences in KRAS protein levels. This proved that some translational programs, such as overexpression of proliferative tRNAs, have the ability to enhance the protein synthesis of oncogenes, including KRAS [47].

A wide range of data has indicated the fundamental importance of ribosomal biogenesis and its relationship with cell proliferation in many aspects of malignant transformations [48]. A series of rare inherited disorders leading to the production of altered ribosomes (so-called ribosomopathies) have even been characterized by a strong risk of cancer onset [49]. An imbalance in the ribosome biogenesis rate via an increase in ribosomal DNA transcription or an alteration in mature rRNA or ribosomal protein production may ultimately lead to the inactivation of p53 through different mechanisms [50]. As a consequence of $p 53$ repression, acquisition of cellular phenotypic changes characteristic of epithelialmesenchymal transition (EMT) results in increased cell invasiveness. In addition, it has been reported that nuclear epithelial cell transforming sequence 2 (ECT2) with GEF activity is required for $K R A S-p 53$ lung tumorigenesis in vivo. ECT2-dependent ribosomal DNA transcription and activation of rRNA synthesis ultimately lead to neoplastic transformation [51]. In addition, nuclear and nucleolar superoxide dismutase are essential for lung cancer cell proliferation through interaction with the PeBoW complex and regulation of pre-rRNA maturation [52].

The RNA components of the spliceosome, uridine-rich (U) snRNAs, can regulate tissue-specific and cancer-specific alternative splicing [53]. Notably, recurrent mutations in U1 snRNA, as one of the most abundant noncoding RNAs, have been recently identified in multiple cancer types and play an important role in the splicing of pre-mRNAs [54]. Collectively, these studies indicate that abnormalities in U1 snRNA and alternative splicing of pre-mRNA are emerging as potentially important drivers of cancer $[54,55]$. An alternative mechanism underlying changes in the U1 levels in alternations of cancer gene expression is changes in $3^{\prime}$-untranslated region (UTR) length, leading to the removal of miRNA binding sites. U1 overexpression lengthens the 3'UTR of KRAS to include a miRNA let-7 binding site with tumor-suppressive activity [56].

snoRNAs are conserved noncoding RNAs responsible for ribonucleoprotein guidance in cells for RNA posttranscriptional modification [57]. A study on the characterization of small snoRNAs in cancer identified an unexpected role for specific snoRNAs in the modulation of KRAS-driven carcinogenesis [58]. A human protein microarray screen discovered SNORD50A and SNORD50B as two snoRNAs that bind to KRAS. The results showed that loss of SNORD50A and SNORD50B expression enhances the amount of GTP-bound and active KRAS, leading to hyperactivated RAS-ERK1/ ERK2 signaling [58]. The soluble NSF attachment protein receptor (SNARE) protein superfamily, which is critical for membrane fusion, is responsible for the vesicular transport that is essential for KRAS trafficking to the plasma membrane and active signaling [59]. In 2019, Che et al. found that the SNORD50A/B snoRNAs, as antagonists of SNAP23, SNAP29, and VAMP3 SNARE proteins, inhibit the process of KRAS localization to the membrane [59].

circRNAs constitute a distinct type of endogenous abundant noncoding RNA with a closed-loop structure and have been found to be overexpressed in cancers [60]. Strikingly, similar to
IncRNAs, circRNAs have the potential to act as oncogenes or tumor suppressors, possibly by acting as sponges for miRNAs. Gorospe et al. found that circPVT1, as a circRNA, regulated the availability of let-7 miRNA, a well-characterized tumor suppressor with a target site on KRAS mRNA. This suggests that circPVT1, whose expression is elevated in dividing cells and downregulated in senescent cells, can be considered a KRAS-related circRNA that acts by sponging let-7 [61]. Other results showed that a circRNA derived from Golgi glycoprotein 1 mRNA regulates $K R A S$ expression and then promotes colorectal cancer development by targeting miR-622 [62].

Many studies have presented remarkable details of systematic alterations in the form of noncoding RNAs, such as miRNAs, IncRNAs, snRNAs, and circRNAs, with impacts on multiple facets of tumorigenesis.

\section{KRAS-RELATED LNCRNAS IN SOLID TUMORS}

Aberrant regulation of oncogenes, tumor suppressor genes, and miRNA genes are crucial in the pathogenesis of cancer. These alterations are sequential multistep processes that can ultimately contribute to malignant transformation [63]. The crucial roles of miRNAs in various biological processes, such as cell proliferation, tumor initiation, EMT, and tumor invasion, are directly related to malignancy [64]. Several studies have identified many tumor suppressor miRNAs targeting the KRAS oncogene in human cancers, which affect cancer-associated cellular and molecular mechanisms $[65,66]$. Notably, research progress on the interactions between IncRNAs and miRNAs in human cancer has introduced an extra layer of complexity in the miRNA-target interaction network [31]. With the development of the analysis of regulatory networks, differential expression, and signaling pathways, IncRNAs have emerged as crucial regulators in various biological processes [67, 68].

In this review, we mainly focus on confirmed KRAS-related IncRNAs whose oncogenic roles as suppressors of KRAS-targeting miRNAs have been verified (Fig. 2). These IncRNAs act as molecular sponges of KRAS-targeting miRNAs, most likely contributing to KRAS upregulation. We also summarize a large number of IncRNAs potentially capable of regulating KRAS, possibly through sponging of previously recognized KRAS-targeting miRNAs (Fig. 2) [31].

\section{CONFIRMED KRAS-RELATED LNCRNAS \\ MALAT1}

MALAT1, which was first identified in lung cancer, plays an important role in the pathogenesis of various human diseases, such as cancer [69-71] and autoimmune and inflammatory diseases [72]. MALAT1 behaves as an oncogene in the initiation and progression of many cancers $[73,74]$. MALAT1, as a molecular sponge of miR-217, an inhibitor of KRAS [75], promotes KRAS signaling in pancreatic ductal adenocarcinoma (PDAC) [76]. In this regard, knockdown of MALAT1 results in a significant reduction in MEK and ERK $1 / 2$ phosphorylation by attenuating KRAS protein expression, emphasizing the role of MALAT1 in protecting KRAS mRNA from repression by miR-217 [76]. Moreover, miR-1 has been shown to suppress breast cancer development by downregulating KRAS and MALAT1 transcription, which emphasizes the potential role of miR-1 as a tumor-suppressive miRNA and MALAT1 as an oncogenic IncRNA via the regulation of KRAS [66].

\section{MIR31HG}

MIR31HG is a IncRNA with 2166 nucleotides that originates from the intronic region of the Harbi1 gene and is responsible for coding miR-31. MIR31HG is markedly upregulated in cancer tissues, with potential roles in cancer initiation, progression, and metastasis. It was confirmed that MIR31HG facilitates esophageal squamous cell carcinoma cell proliferation and functions as a 


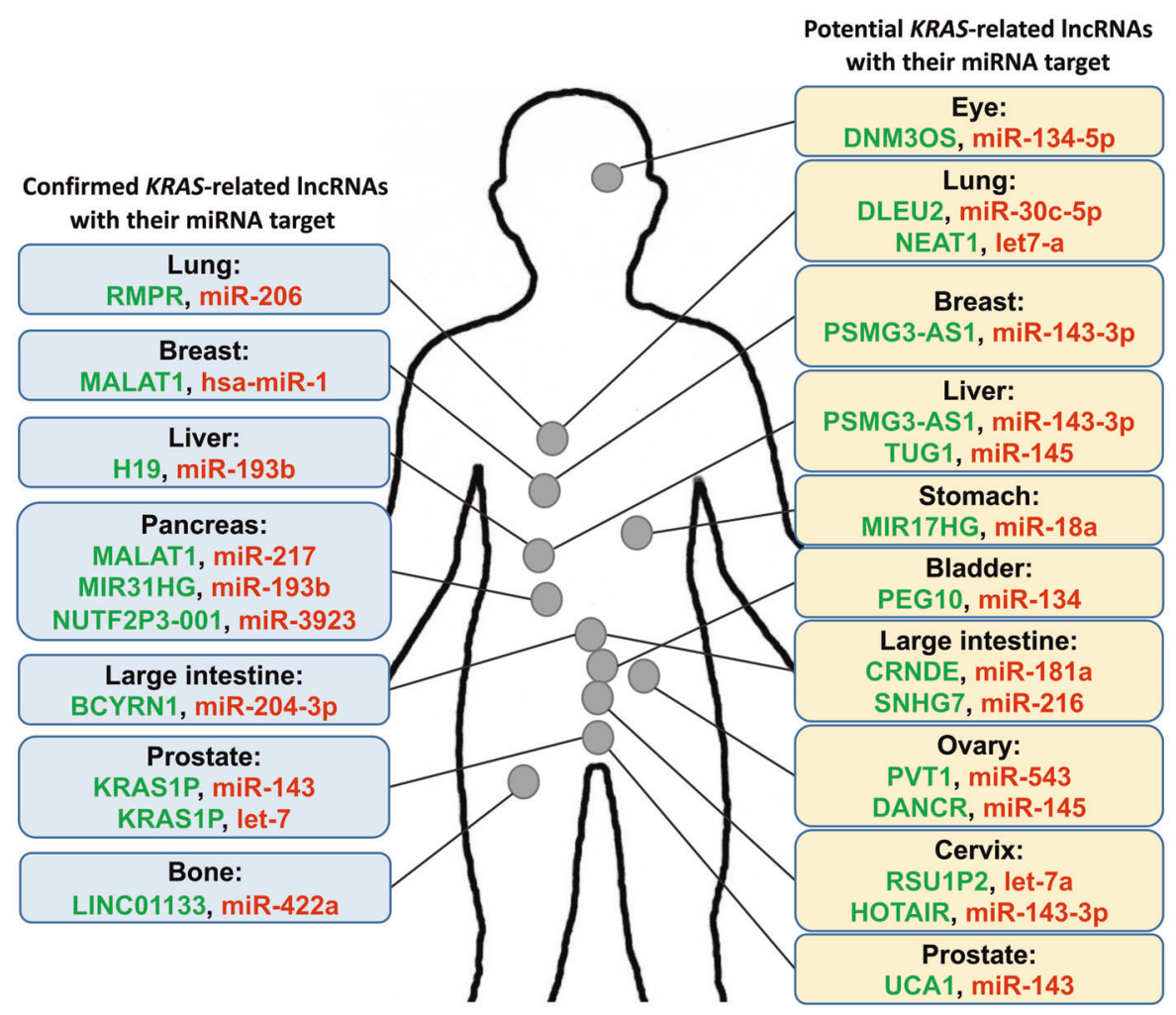

Fig. 2 Lists of the confirmed (left) and potential (right) KRAS-related IncRNAs in different tissues. IncRNAs are presented in green, indicating their upregulation as oncogenic regulators in carcinogenesis. miRNAs with tumor suppressor activity are presented in red, indicating their repression due to the sponging effect of IncRNAs in malignancies. The left and right panels illustrate the confirmed and potential KRAS-related IncRNAs as well as their miRNAs, respectively (Supplementary Table S1).

ceRNA by sponging miR-34a, allowing upregulation of c-Met [77]. MIR31HG inhibits oncogene-induced cell senescence by regulating transcription of the tumor suppressor p16 (INK4A) [78]. The interaction of the MIR31HG transcript with the genomic regions of INK4A and MIR31HG contributes to the recruitment of polycombgroup protein complexes and then the repression of INK4A. In addition, SP1-induced MIR31HG was found to be significantly upregulated in NSCLC tissues and cell lines, which promotes cell migration and invasion by sponging miR-214 [79]. It has been reported that miR-193b is able to directly target MIR31HG, resulting in cancer progression by counteracting miR-193b in pancreatic cancer [80]. Based on the significant role of the KRAS mutation in pancreatic cancer, these results confirm the potential role of MIR31HG in the malignant transformation of different tumors, including KRAS-driven pancreatic cancer.

\section{KRAS1P}

KRAS1P is considered as a pseudogene of KRAS. Its expression is amplified in most cancers with mutated KRAS, which indicates a positive correlation between these genes. The transcript levels of KRAS and KRAS1P correlate directly in prostate cancer, neuroblastoma, retinoblastoma, and hepatocellular carcinoma (HCC), which illustrates a proto-oncogenic role of KRAS1P in cancer [8183]. While the detailed mechanism by which KRAS1P regulates KRAS as a pseudogene-derived noncoding RNA has not been well recognized, its activity as a sponge for miRNAs that bind to the $3^{\prime}$ UTR of KRAS has been proposed [84]. Two studies have reported the possible role of KRAS1P as a ceRNA with binding sites for some KRAS-targeting miRNAs, such as miR-143 and the let-7 miRNA family $[85,86]$. Thus, KRAS1P can potentially act as an oncogenic IncRNA to inhibit degradation of the KRAS transcript [84].

\section{BCYRN1}

BCYRN1 is a newly identified brain cytoplasmic IncRNA of 200 nucleotides, which is transcribed from human chromosome $2 \mathrm{p} 21$. The high expression of BCYRN1 in various tumor cell lines suggests the role of BCYRN1 as an oncogenic IncRNA [87, 88]. In gastric cancer tissues, it is associated with tumor depth, lymph node metastasis, cell proliferation, cell cycle progression, migration, and invasion [89]. BCYRN1 is upregulated in colorectal cancer (CRC) tissues, which is related to tumor growth and advanced pathological stages via NPR3 overexpression [90]. Moreover, the promotion of glycolysis and tumor progression in non-small cell lung cancer (NSCLC) are observed as the result of BCYRN1 overexpression [91]. High BCYRN1 expression induces glycolysis through the repression of miR-149 and upregulation of PKM2 as the target of miR-149. Strikingly, as a ceRNA, BCYRN1 affects the development of CRC via regulation of the miR-204$3 \mathrm{p} / K R A S$ axis [92]. Therefore, negative regulation of KRAS by miR-204-3p suggests BCYRN1 as another confirmed KRASrelated IncRNA.

\section{NUTF2P3-001}

Overexpression of NUTF2P3-001 in pancreatic cancer and chronic pancreatitis tissues is positively correlated with cancer cell characteristics, such as tumor size and distant metastasis [93]. It was reported that NUTF2P3-001, as an oncogenic IncRNA, competes with the $3^{\prime}$ UTR of KRAS mRNA for binding to miR3923. In addition, downregulation of NUTF2P3-001 inhibits the viability, proliferation, and invasion of pancreatic cancer cells and contributes to a decrease in KRAS expression [93]. Hence, these data provide an alternative IncRNA-mediated regulatory mechanism for the tumor oncogene KRAS. 


\section{RMRP}

RMRP IncRNA is widely expressed in different human and mouse tissues [94]. Previous studies have indicated that the expression of RMRP is dysregulated in gastric cancer [95]. Suppression of miR-206 by RMRP positively modulates Cyclin D2 expression and cell cycle progression, which provides us with a better understanding of the mechanism underlying RMPR carcinogenesis [96]. Furthermore, ectopic expression of RMRP was observed to promote cell proliferation, colony formation, and invasion in lung adenocarcinoma [97]. It was indicated that miR-206 acts as a tumor suppressor miRNA in oral squamous cell carcinoma by directly targeting KRAS [98]. Inhibition of miR-206 by RMRP was demonstrated to result in overexpression of KRAS, FMNL2, and SOX9 in lung adenocarcinoma [99], confirming RMPR as one of the KRAS-related IncRNAs.

\section{H19}

H19, with both oncogenic and tumor suppressor activities, acts as a double-edged sword via mechanisms such as miRNA sponging [100]. The let-7 family miRNAs that control human RAS oncogene expression are often downregulated in human cancers $[86,101,102]$. H19 possesses both canonical and noncanonical binding sites for the let-7 family of miRNAs, which plays predominant roles not only in cancer but also in development and metabolism [103]. H19 promotes pancreatic cancer metastasis by inhibiting let-7 suppression on its target HMGA2-mediated EMT in PDACs $[100,104]$. Considering the role of let-7 in targeting KRAS, $\mathrm{H} 19$ may influence KRAS expression levels in PDAC. To confirm other sponging effects of $\mathrm{H} 19, \mathrm{H} 19$ overexpression exerted proangiogenic effects, possibly by downregulating miR-181a and inducing the JNK and AMPK signaling pathways to facilitate angiogenesis [30]. Considering the tumor-suppressive effect of miR-181a via downregulation of KRAS and the role of the KRAS mutation in vascular malformations, it is assumed that $\mathrm{H} 19$ has an indirect effect on KRAS upregulation [105, 106]. This can also be mediated by miR-193b, another KRAS-regulating miRNA [107]. Overexpression of $\mathrm{H} 19$ has been shown to attenuate miR-193bmediated inhibition of multiple driver oncogenes, including $E G F R$, KRAS, PTEN, IGF1R, and MAPK1, suggesting that InCRNA H19 serves as a KRAS regulator through miR-193b sponging [108].

\section{LINC01133}

LINC01133, with a length of 1154 nucleotides, is located on chromosome 1q23.2 and was first reported to be involved in CRC and NSCLC [109, 110]. A positive correlation has been found between high LINC01133 expression and poor prognosis in patients. LINC01133 downregulation leads to the repression of proliferation and invasion of lung cancer cells [111]. Nevertheless, other studies have shown low LINC01133 expression in CRC and breast cancer tissues $[112,113]$. Therefore, it can be concluded that the expression levels of LINC01133 vary among various types of cancer, suggesting that there is a tissue-specific regulation of its expression that may be directly related to its function. Other results showed that LINC01133 aggravates the proliferation, migration, and invasion of osteosarcoma by sponging miR-422a, which targets KRAS, exerting antitumor effects [114, 115].

\section{SLCO4A1-AS1}

The role of SLCO4A1-AS1 in the tumorigenesis of CRC has been demonstrated in several studies, confirming its upregulation in CRC tissues and its relation with poor prognosis and tumor metastasis $[116,117]$. SLCO4A1-AS1 has been reported to serve as an oncogenic IncRNA in CRC by activating the WNT/ $\beta$-catenin signaling pathway [117]. The oncogenic role of SLCO4A1-AS1 in CRC promotion has been attributed to the stabilization of SLCO4A1, a transmembrane protein with sodium-independent organic anion transporter activity. In addition, the axis of the SLCO4A1-AS1/miR-508-3p/PARD3 autophagy pathway has been proposed as another carcinogenic mechanism of SLCO4A1-AS1 in the development of CRC through a sponging effect [116]. SLCO4A1-AS1 knockdown in HCT116 and SW480 cells led to the downregulation of EGFR, KRAS, BRAF, and MAP3K1 expression [118]. Therefore, SLCO4A1-AS1 can be considered as a KRASrelated IncRNA. However, the corresponding miRNA has not yet been identified.

\section{POTENTIAL KRAS-RELATED LNCRNAS}

On the basis of the significant role of KRAS oncogenic mutations, many miRNAs that target KRAS have been discovered in many human cancer tissues $[119,120]$. The inhibitory effect of miRNAs on KRAS expression led us to search for miRNAs that are sponged by oncogenic IncRNAs to find potential KRAS-related IncRNAs. Therefore, a review of the previously recognized KRAS-targeting miRNAs helps us to predict some oncogenic IncRNAs with sponging effects, which may participate in the regulation of $K R A S$. To identify potential KRAS-related IncRNAs, two steps were taken. In the first step, a collection of miRNAs that target KRAS were identified. Second, an extensive literature study was performed to determine IncRNAs with sponging effects on the miRNAs (Fig. 2). For example, a significant role of miR-143 in the inhibition of KRAS translation was confirmed to contribute to the suppression of cell growth [85]. In this regard, other supporting documents showed the interaction of PSMG3-AS1 IncRNA as a sponge with miR-143-3p in HCC and breast cancer tissues $[16,121]$. According to the targeting of KRAS by miR-143 and the sponging effect of PSMG3-AS1 on this miRNA, it can be assumed that PSMG3-AS1 can be a potential KRAS-associated IncRNA. Similarly, miR-181a is a known miRNA with the ability to target KRAS mRNA. With this information, IncRNA CRNDE, whose sponging effect on miR-181a was previously confirmed, can be considered one of the other potential KRAS-related IncRNAs [122]. Therefore, a thorough understanding of the plethora of tumor suppressor miRNAs contributing to KRAS-targeting and its downregulation provides mechanistic insight into discovering potential KRAS-related oncogenic IncRNAs that act as molecular sponges. Accordingly, there is a large number of potential KRASrelated IncRNAs sponging the KRAS-targeting miRNAs (Fig. 2; Supplementary Table S1).

\section{RAS-RELATED LNCRNAS ASSOCIATED WITH LEUKEMIA}

Leukemia, as a heterogeneous group of malignant neoplasms in the hematopoietic system, is classified on the basis of its clinical behavior and histological origin. Although leukemia is a common malignant cancer of the hematopoietic system, its mechanism of pathogenesis has not been fully elucidated [123]. One of the main causes of this malignancy is related to acquired and infrequently inherited genetic alterations [124]. Moreover, epigenetic alterations, such as heritable and reversible changes, can also lead to some malignant behaviors, such as cancer relapse. For instance, as well-studied leukemia, acute myeloid leukemia (AML) is a typical consequence of these abnormalities and gene mutations [125]. In addition to these valuable efforts, an urgent need to elucidate the mechanism of cancer malignancy triggered the researchers to search for new molecular systems, including regulatory transcripts such as miRNAs and IncRNAs.

Oncogenic RAS mutations are highly prevalent in hematopoietic malignancies and are associated with poor survival [126]. While somatic mutations, such as KRAS mutations, cause a series of downstream secondary alterations in the transcriptome of cancer cells, evidence showing the role of IncRNAs in the pathophysiology of hematological malignancies has drastically increased in the last decade [127]. Therefore, understanding the role of KRAS mutations in large-scale alterations in the transcriptional profiles of leukemia cells, including the dysregulation of IncRNA 
expression, provides more details on the pathogenic mechanisms. In this regard, the results of a pairwise analysis study comparing patients with KRAS mutations showed 26 differentially expressed IncRNAs (17 upregulated and 9 downregulated) compared to juvenile myelomonocytic leukemia (JMML) patients without this mutation [128]. Other differentially expressed RNAs between JMML patients and normal bone marrow controls revealed that the expression of 29 (19 up- and 10 downregulated) IncRNAs was dysregulated in the subgroup of KRAS-mutant patients with overexpressed Inc-ACOT9-1 [129]. IncRNA MORRBID regulates the lifespan of short-lived myeloid cells in response to extracellular pro-survival signals through the suppression of the pro-apoptotic gene BCL2L11 (also known as BIM) [130]. The high expression of MORRBID accompanied by KRAS and NRAS mutations is associated with poor overall survival of JMML patients [131].

Although the exact mechanism by which KRAS-related IncRNAs function in leukemia has not been elucidated, the sponging effect on miRNAs can be considered one of the regulatory procedures. Wang and colleagues demonstrated the role of MALAT1 in sponging miR-101 to inhibit its interaction with the 3UTR of its target mRNA, myeloid cell leukemia 1 (MCL1). This competition between MALAT1 and MCL1 causes a decrease in MCL1 expression and a consequent increase in drug resistance in lung cancer [132]. In addition to the contribution of IncRNAs in leukemogenesis, recent studies on the role of IncRNAs as biomarkers in the diagnosis, prognosis, and therapeutic response have emphasized IncRNAs as essential regulatory factors in leukemia patients [133-135].

\section{IncRNAs as therapeutic targets}

IncRNAs are key regulators of gene expression and act through different mechanisms, including genomic imprinting, epigenetic regulation, mRNA and protein stability regulation, protein sequestration, miRNA sponging, protein translation regulation, and alternative splicing. Therefore, not only sponging effects but also other mechanisms are involved in gene regulation by IncRNAs, which provides the possible application of extensive therapeutic strategies [136].

With rapid developments in high-throughput screening methods and bioinformatics, large numbers of cancer-related genes and their associated regulatory IncRNAs will be discovered in the near future [137-139]. Considering the critical roles of IncRNAs in malignancies, IncRNA-based therapeutics may represent promising approaches in cancer treatment through novel technologies $[140,141]$. Antisense oligonucleotides (ASOs), which may form a DNA-RNA structure with their target RNA through base pairing rules, could be exploited as promising tools for targeting oncogenic IncRNAs [142]. Aptamers are specific structures in the form of oligonucleotides or peptide molecules that possess the ability to bind specifically and structurally to the desired target, such as IncRNA, and prevent the interactions of the IncRNA with its corresponding targets [136]. The CRISPR/Cas9 genome editing technique, a technology for the specific DNA modification of targeted genes, has been found to be a successful approach to silence the transcription of many carcinogenic IncRNAs [143]. Although the rapid development of a new generation of geneediting tools, such as ASOs or CRISPR/Cas9-based therapy, has already shown the feasibility of gene-editing for cancer treatment, their off-target events or unstable efficiency originating from the spatiotemporal specificity of IncRNAs should also be evaluated for further clinical applications [14]. Neutralizing targeted IncRNAs by exogenous double-stranded RNA via RNA interference (RNAi) transfection is an alternative strategy that has shown some significant results due to its specificity [144]. Despite its specificity, the RNAi method efficiency is transient due to the natural instability of RNA molecules, which necessitates solid experimental analysis to confirm the practicability of this technology [145]. In contrast to oncogenic IncRNAs, some IncRNAs with tumor suppressor activity, such as CR749391 and LET, are known to be expressed at low levels in tumors [146, 147]. Thus, induction of these IncRNAs could be a possible therapeutic approach for cancer treatment. For example, viral transfection, as the main method for plasmid transmission to the target site, could be applied to transfect exogenously synthesized tumor suppressor IncRNA plasmids into cancer cells to upregulate the expression of corresponding IncRNAs. This IncRNA-based strategy could be investigated for cancer treatment; however, solid experimental analysis is required to validate the feasibility and practicability of this strategy [14]. Aside from the fact that IncRNAs themselves could serve as possible therapeutic targets, recent documents have proven the utility of peptides/proteins encoded by IncRNAs as other potential targets [148]. IncRNAs are known as RNA molecules that do not encode proteins, but recent findings have shown that peptides/proteins encoded by IncRNAs do indeed exist and surprisingly have tumorigenic effects [148]. Therefore, peptides/proteins encoded by IncRNAs might be hidden oncopeptides/oncoproteins representing promising drug targets for treating tumor growth [148]. On the other hand, some proteins encoded by IncRNAs have tumor-suppressive effects that inhibit the carcinogenesis of oncoproteins such as KRAS [38]. Taken together, these findings suggest that IncRNAs could serve as novel therapeutic targets for cancer therapy.

\section{CONCLUSION AND PERSPECTIVE}

Approximately $25 \%$ of all human cancers have oncogenic mutations in the RAS family of oncogenes, most frequently the KRAS gene, resulting in the aberrant activation of RAS proteins and consequently their downstream pathways and leading to malignant transformation. To date, diverse therapeutic approaches have been used to interfere with mutant KRASmediated signaling. Although KRAS proto-oncogene mutations are responsible for the conversion of KRAS to its oncoprotein form with increased activity, suppression of mutant KRAS gene expression could be an approach to inhibit oncoprotein production. In this review, we focused on the sponging effect as a strategy for KRAS downregulation, considering the established roles of both miRNAs and IncRNAs. The fact that the majority of IncRNAs are expressed in a highly cell- or tissue-specific manner makes them effective therapeutic targets for cancer treatment. However, many questions remain to be addressed. How many IncRNAs are functionally and clinically relevant for KRAS-driven cancers? How can we develop systematic genomic and functional approaches to understand the role of IncRNAs in the initiation, progression, and alternative metastasis of KRAS-mutant cancers? How can we integrate patient genomic and transcriptomic data with KRAS mutations to establish a IncRNA discovery pipeline to drive preclinical studies? Finally, how does a tissue-specific expression of IncRNAs provide therapeutic candidates for tissues with a higher frequency of KRAS mutation? In addition to the questions above, the authors of this review present some suggestions for future studies concerning IncRNAs as therapeutic targets. More oncogenic IncRNAs with sponging effects on other tumor-suppressive miRNAs that target KRAS or its downstream effectors should be discovered. Proteins/peptides encoded by IncRNAs and their oncogenic or tumor-suppressing effects should be investigated. The ability to target KRAS-related oncogenic IncRNAs through various methods, such as nucleic acid-based drugs, gene-editing methods, small molecule inhibitors, miRNA mimics, catalytic degradation of IncRNAs by ribozymes, targeting IncRNA secondary and tertiary structures, and synthetic IncRNA mimics, must be studied. More importantly, further characterization of interactions between oncogenic IncRNAs and associating proteins, which form ribonucleoprotein complexes and could be involved in KRAS signaling, may lead to the identification of novel 
therapeutic targets and the development of new anti-KRAS drugs. Hopefully, the increased success rate of nucleic acid therapeutics provides an outstanding opportunity to discover IncRNAs as viable candidates for therapeutic targets in KRAS-dependent malignant transformation.

\section{REFERENCES}

1. Simanshu DK, Nissley DV, McCormick F. RAS proteins and their regulators in human disease. Cell. 2017;170:17-33.

2. Fruman DA, Chiu H, Hopkins BD, Bagrodia S, Cantley LC, Abraham RT. The PI3K pathway in human disease. Cell. 2017;170:605-35.

3. Yuan TL, Amzallag A, Bagni R, Yi M, Afghani S, Burgan W, et al. Differential effector engagement by oncogenic KRAS. Cell Rep. 2018;22:1889-902.

4. Hanahan D, Weinberg RA. Hallmarks of cancer: the next generation. Cell. 2011;144:646-74.

5. Scheffzek K, Ahmadian MR, Kabsch W, Wiesmüller L, Lautwein A, Schmitz F, et al. The Ras-RasGAP complex: structural basis for GTPase activation and its loss in oncogenic Ras mutants. Science. 1997;277:333-9.

6. Scheffzek K, Ahmadian MR, Wittinghofer A. GTPase-activating proteins: helping hands to complement an active site. Trends Biochem Sci. 1998;23:257-62.

7. McCormick F. K-Ras protein as a drug target. J Mol Med (Berl). 2016;94:253-8. https://doi.org/10.1158/1078-0432.

8. Ryan MB, Corcoran RB. Therapeutic strategies to target RAS-mutant cancers. Nat Rev Clin Oncol. 2018;15:709-20.

9. Saliani $M$, Jalal $R$, Ahmadian MR. From basic researches to new achievements in therapeutic strategies of KRAS-driven cancers. Cancer Biol Med. 2019;16:435-61.

10. Moore AR, Rosenberg SC, McCormick F, Malek S. RAS-targeted therapies: is the undruggable drugged? Nat Rev Drug Discov. 2020;19:533-52.

11. Stalnecker CA, Der CJ. RAS, wanted dead or alive: advances in targeting RAS mutant cancers. Sci Signal. 2020;13:eaay6013.

12. Misale $S$, Yaeger R, Hobor S, Scala E, Janakiraman M, Liska D, et al. Emergence of KRAS mutations and acquired resistance to anti-EGFR therapy in colorectal cancer. Nature. 2012;486:532-6.

13. Goodall GJ, Wickramasinghe VO. RNA in cancer. Nat Rev Cancer. 2021;21:22-36.

14. Jiang $M-C$, Ni J-J, Cui W-Y, Wang B-Y, Zhuo W. Emerging roles of IncRNA in cancer and therapeutic opportunities. Am J Cancer Res. 2019;9:1354-66.

15. Bhan A, Soleimani $M$, Mandal SS. Long noncoding RNA and cancer: a new paradigm. Cancer Res. 2017;77:3965-81.

16. Cui $Y$, Fan $Y$, Zhao G, Zhang Q, Bao $Y$, Cui $Y$, et al. Novel IncRNA PSMG3-AS1 functions as a miR-143-3p sponge to increase the proliferation and migration of breast cancer cells. Oncol Rep. 2020;43:229-39.

17. Dai Q, Deng J, Zhou J, Wang Z, Yuan X-F, Pan S, et al. Long non-coding RNA TUG1 promotes cell progression in hepatocellular carcinoma via regulating miR216b-5p/DLX2 axis. Cancer Cell Int. 2020;20:8.

18. Domvri K, Petanidis S, Anestakis D, Porpodis K, Bai C, Zarogoulidis P, et al. Exosomal IncRNA PCAT-1 promotes Kras-associated chemoresistance via immunosuppressive miR-182/miR-217 signaling and p27/CDK6 regulation. Oncotarget. 2020;11:2847-62.

19. Fernandes JCR, Acuña SM, Aoki Jl, Floeter-Winter LM, Muxel SM. Long noncoding RNAs in the regulation of gene expression: physiology and disease. NonCoding RNA. 2019;5:17.

20. Lin X, Yang F, Qi X, Li Q, Wang D, Yi T, et al. LncRNA DANCR promotes tumor growth and angiogenesis in ovarian cancer through direct targeting of miR-145. Mol Carcinog. 2019;58:2286-96.

21. Liu C, Hou J, Shan F, Wang L, Lu H, Ren T. Long non-coding RNA CRNDE promotes colorectal carcinoma cell progression and paclitaxel resistance by regulating miR-126-5p/ATAD2 axis. Onco Targets Ther. 2020;13:4931-42.

22. López-Urrutia E, Bustamante Montes LP, Ladrón de Guevara Cervantes D, PérezPlasencia C, Campos-Parra AD. Crosstalk between long non-coding RNAs, MicroRNAs and mRNAs: deciphering molecular mechanisms of master regulators in cancer. Front oncol. 2019;9:669.

23. Wu H, Wei M, Jiang X, Tan J, Xu W, Fan X, et al. IncRNA PVT1 promotes tumorigenesis of colorectal cancer by stabilizing miR-16-5p and interacting with the VEGFA/VEGFR1/AKT axis. Mol Ther - Nucleic Acids. 2020;20:438-50.

24. Zhu K, Ding $H, Y u$ A, Liao Z, Fu Z, Hong $Y$, et al. Co-targeting of EGFR by coexpressed miRNA-193a-3p/-5p in lung cancer. ExRNA. 2019;1:29.

25. Motti ML, Minopoli M, Carluccio GD, Ascierto PA, Carriero MV. MicroRNAs as key players in melanoma cell resistance to MAPK and immune checkpoint inhibitors. Int J Mol Sci. 2020;21:4544.

26. Kolenda T, Guglas K, Kopczyńska M, Sobocińska J, Teresiak A, Bliźniak R, et al. Good or not good: role of miR-18a in cancer biology. Rep Pract Oncol Radiother. 2020;25:808-19.
27. Kolenda T, Przybyła W, Teresiak A, Mackiewicz A, Lamperska KM. The mystery of let-7d-a small RNA with great power. Contemp Oncol. 2014;18:293.

28. Yuan J, Tan L, Yin Z, Zhu W, Tao K, Wang G, et al. MIR17HG-miR-18a/19a axis, regulated by interferon regulatory factor-1, promotes gastric cancer metastasis via Wnt/ $\beta$-catenin signalling. Cell Death Dis. 2019;10:454.

29. Zhang J, Huang J, Chen W, Hu Z, Wang X. miR-143-3p targets IncRNA PSMG3AS1 to inhibit the proliferation of hepatocellular carcinoma cells. Cancer Manag Res. 2020;12:6303.

30. Zhu A, Chu L, Ma Q, Li Y. Long non-coding RNA H19 down-regulates miR-181a to facilitate endothelial angiogenic function. Artif Cells Nanomed Biotechnol. 2019;47:2698-705.

31. Sun B, Liu C, Li H, Zhang L, Luo G, Liang $S$, et al. Research progress on the interactions between long non-coding RNAs and microRNAs in human cancer (Review). Oncol Lett. 2020;19:595-605.

32. Lewis BP, Burge CB, Bartel DP. Conserved seed pairing, often flanked by adenosines, indicates that thousands of human genes are microRNA targets. Cell. 2005;120:15-20.

33. Li Z, Zhao W, Wang M, Zhou X. The role of long noncoding RNAs in gene expression regulation. Gene Expression Profiling in Cancer. 2019. https://www. intechopen.com/chapters/64307.

34. He R-Z, Luo D-X, Mo Y-Y. Emerging roles of IncRNAs in the post-transcriptional regulation in cancer. Genes Dis. 2019;6:6-15.

35. Kansara S, Pandey V, Lobie PE, Sethi G, Garg M, Pandey AK. Mechanistic involvement of Long non-coding RNAs in Oncotherapeutics resistance in triplenegative breast cancer. Cells. 2020;9:1511.

36. Morgan RK, Batra $\mathrm{H}$, Gaerig VC, Hockings J, Brooks TA. Identification and characterization of a new G-quadruplex forming region within the kRAS promoter as a transcriptional regulator. Biochim Biophys Acta. 2016;1859:235-45.

37. Wu R, Li L, Bai Y, Yu B, Xie C, Wu H, et al. The long noncoding RNA LUCAT1 promotes colorectal cancer cell proliferation by antagonizing Nucleolin to regulate MYC expression. Cell Death Dis. 2020;11:908.

38. Xu W, Deng B, Lin P, Liu C, Li B, Huang Q, et al. Ribosome profiling analysis identified a KRAS-interacting microprotein that represses oncogenic signaling in hepatocellular carcinoma cells. Sci China Life Sci. 2020;63:529-42.

39. Shi L, Magee P, Fassan M, Sahoo S, Leong HS, Lee D, et al. A KRAS-responsive long non-coding RNA controls microRNA processing. Nat Commun. 2021;12:1-19.

40. Zhang D, Zhang G, Hu X, Wu L, Feng Y, He S, et al. Oncogenic RAS regulates long noncoding RNA Orilnc1 in human cancer. Cancer Res. 2017;77:3745-57.

41. Balatti V, Pekarsky Y, Croce CM. Role of the tRNA-derived small RNAs in cancer: new potential biomarkers and target for therapy. Adv Cancer Res. 2017;135:173-87.

42. Goodarzi H, Nguyen HC, Zhang S, Dill BD, Molina H, Tavazoie SF. Modulated expression of specific tRNAs drives gene expression and cancer progression. Cell. 2016;165:1416-27.

43. Pekarsky Y, Balatti V, Palamarchuk A, Rizzotto L, Veneziano D, Nigita G, et al. Dysregulation of a family of short noncoding RNAs, tsRNAs, in human cancer. Proc Natl Acad Sci. 2016;113:5071-6.

44. Zhang Y, Qian H, He J, Gao W. Mechanisms of tRNA-derived fragments and tRNA halves in cancer treatment resistance. Biomark Res. 2020;8:52.

45. Balatti V, Nigita G, Veneziano D, Drusco A, Stein GS, Messier TL, et al. tsRNA signatures in cancer. Proc Natl Acad Sci. 2017;114:8071-6.

46. Rimawi MF, De Angelis C, Contreras A, Pareja F, Geyer FC, Burke KA, et al. Low PTEN levels and PIK3CA mutations predict resistance to neoadjuvant lapatinib and trastuzumab without chemotherapy in patients with HER2 over-expressing breast cancer. Breast Cancer Res Treat. 2018;167:731-40.

47. Benisty $H$, Weber M, Hernandez-Alias X, Schaefer MH, Serrano L. Proliferation specific codon usage facilitates oncogene translation. Proc Natl Acad Sci U S A. 2020;117:30848-56.

48. Penzo $M$, Montanaro $L$, Treré $D$, Derenzini $M$. The ribosome biogenesis-cancer connection. Cells. 2019:8:55.

49. Narla A, Ebert BL. Ribosomopathies: human disorders of ribosome dysfunction. Blood, J Am Soc Hematol. 2010;115:3196-205.

50. Donati G, Bertoni S, Brighenti E, Vici M, Trere D, Volarevic S, et al. The balance between rRNA and ribosomal protein synthesis up-and downregulates the tumour suppressor p53 in mammalian cells. Oncogene. 2011:30:3274-88.

51. Justilien V, Ali SA, Jamieson L, Yin N, Cox AD, Der CJ, et al. Ect2-dependent rRNA synthesis is required for KRAS-TRP53-driven lung adenocarcinoma. Cancer Cell. 2017;31:256-69.

52. Wang $X$, Zhang $H$, Sapio $R$, Yang J, Wong J, Zhang $X$, et al. SOD1 regulates ribosome biogenesis in KRAS mutant non-small cell lung cancer. Nat Commun. 2021;12:2259.

53. Dvinge $\mathrm{H}$, Guenthoer J, Porter PL, Bradley RK. RNA components of the spliceosome regulate tissue-and cancer-specific alternative splicing. Genome Res. 2019;29:1591-604. 
54. Shuai S, Suzuki H, Diaz-Navarro A, Nadeu F, Kumar SA, Gutierrez-Fernandez A, et al. The U1 spliceosomal RNA is recurrently mutated in multiple cancers. Nature. 2019;574:712-6.

55. Dvinge $\mathrm{H}$, Kim $\mathrm{E}$, Abdel-Wahab O, Bradley RK. RNA splicing factors as oncoproteins and tumour suppressors. Nat Rev Cancer. 2016;16:413.

56. Oh J-M, Venters CC, Di C, Pinto AM, Wan L, Younis I, et al. U1 snRNP regulates cancer cell migration and invasion in vitro. Nat Commun. 2020;11:1.

57. Liang J, Wen J, Huang Z, Chen X-P, Zhang B-X, Chu L. Small nucleolar RNAs: insight into their function in cancer. Front Oncol. 2019;9:587.

58. Siprashvili Z, Webster DE, Johnston D, Shenoy RM, Ungewickell AJ, Bhaduri A, et al. The noncoding RNAs SNORD50A and SNORD50B bind K-Ras and are recurrently deleted in human cancer. Nat Genet. 2016;48:53-8.

59. Che Y, Siprashvili Z, Kovalski JR, Jiang T, Wozniak G, Elcavage L, et al. KRAS regulation by small non-coding RNAs and SNARE proteins. Nat Commun. 2019;10:5118.

60. Vo JN, Cieslik M, Zhang Y, Shukla S, Xiao L, Zhang Y, et al. The landscape of circular RNA in cancer. Cell. 2019;176:869-81.e13.

61. Panda AC, Grammatikakis I, Kim KM, De S, Martindale JL, Munk R, et al. Identification of senescence-associated circular RNAs (SAC-RNAs) reveals senescence suppressor CircPVT1. Nucleic Acids Res. 2017;45:4021-35.

62. Hao S, Qu R, Hu C, Wang M, Li Y. A circular RNA derived from golgi glycoprotein 1 mRNA regulates KRAS expression and promotes colorectal cancer progression by targeting microRNA-622. Onco Targets Ther. 2020;13:12637.

63. Jansson MD, Lund AH. MicroRNA and cancer. Mol Oncol. 2012;6:590-610.

64. Macfarlane L-A, Murphy PR. MicroRNA: biogenesis, function and role in cancer. Curr Genomics. 2010;11:537-61.

65. Kazmi HR, Chandra A, Kumar S, Satyam LK, Gupta A, Nigam J, et al. A let-7 microRNA binding site polymorphism in the KRAS $3^{\prime}$ UTR is associated with increased risk and reduced survival for gallbladder cancer in North Indian population. J Cancer Res Clin Oncol. 2016;142:2577-83.

66. Liu R, Li J, Lai Y, Liao Y, Liu R, Qiu W. Hsa-miR-1 suppresses breast cancer development by down-regulating K-ras and long non-coding RNA MALAT1. Int J Biol Macromolecules. 2015;81:491-7.

67. Huang Q, Yan J, Agami R. Long non-coding RNAs in metastasis. Cancer Metastasis Rev. 2018;37:75-81.

68. Rossi MN, Antonangeli F. LncRNAs: new players in apoptosis control. Int J Cell Biol. 2014;2014:473857.

69. Jiao F, Hu H, Han T, Yuan C, Wang L, Jin Z, et al. Long noncoding RNA MALAT-1 enhances stem cell-like phenotypes in pancreatic cancer cells. Int J Mol Sci. 2015:16:6677-93.

70. Li L, Chen H, Gao Y, Wang YW, Zhang GQ, Pan SH, et al. Long Noncoding RNA MALAT1 promotes aggressive pancreatic cancer proliferation and metastasis via the stimulation of autophagy. Mol Cancer Therapeutics. 2016;15:2232-43.

71. Tang R, Jiang M, Liang L, Xiong D, Dang $Y$, Chen G. Long noncoding RNA MALAT-1 can predict poor prognosis: a meta-analysis. Med Sci Monit. 2016;22:302-9.

72. Yang $\mathrm{H}$, Liang $\mathrm{N}$, Wang $\mathrm{M}$, Fei $\mathrm{Y}$, Sun J, Li Z, et al. Long noncoding RNA MALAT-1 is a novel inflammatory regulator in human systemic lupus erythematosus. Oncotarget. 2017;8:77400-6.

73. Gutschner T, Hämmerle M, Eissmann M, Hsu J, Kim Y, Hung G, et al. The noncoding RNA MALAT1 is a critical regulator of the metastasis phenotype of lung cancer cells. Cancer Res. 2013;73:1180-9.

74. Wei Y, Niu B. Role of MALAT1 as a prognostic factor for survival in various cancers: a systematic review of the literature with meta-analysis. Dis Markers. 2015;2015:164635.

75. Zhao WG, Yu SN, Lu ZH, Ma YH, Gu YM, Chen J. The miR-217 microRNA functions as a potential tumor suppressor in pancreatic ductal adenocarcinoma by targeting KRAS. Carcinogenesis. 2010;31:1726-33.

76. Liu $\mathrm{P}$, Yang $\mathrm{H}$, Zhang J, Peng $\mathrm{X}$, Lu Z, Tong W, et al. The IncRNA MALAT1 acts as a competing endogenous RNA to regulate KRAS expression by sponging miR-217 in pancreatic ductal adenocarcinoma. Sci Rep. 2017;7:5186.

77. Chu J, Jia J, Yang L, Qu Y, Yin H, Wan J, et al. LncRNA MIR31HG functions as a ceRNA to regulate c-Met function by sponging miR-34a in esophageal squamous cell carcinoma. Biomedicine Pharmacother. 2020;128:110313.

78. Montes M, Nielsen MM, Maglieri G, Jacobsen A, Højfeldt J, Agrawal-Singh S, et al. The IncRNA MIR31HG regulates p16 INK4A expression to modulate senescence. Nat Commun. 2015;6:1-15.

79. Dandan W, Jianliang $C$, Haiyan $H$, Hang $M$, Xuedong L. Long noncoding RNA MIR31HG is activated by SP1 and promotes cell migration and invasion by sponging miR-214 in NSCLC. Gene. 2019;692:223-30.

80. Yang H, Liu P, Zhang J, Peng X, Lu Z, Yu S, et al. Long noncoding RNA MIR31HG exhibits oncogenic property in pancreatic ductal adenocarcinoma and is negatively regulated by miR-193b. Oncogene. 2016;35:3647-57.
81. Plantaz D, Mohapatra G, Matthay KK, Pellarin M, Seeger RC, Feuerstein BG. Gain of chromosome 17 is the most frequent abnormality detected in neuroblastoma by comparative genomic hybridization. Am J Pathol. 1997;150:81-9.

82. van der Wal JE, Hermsen MAJA, Gille HJP, Schouten-Van Meeteren NYN, Moll AC, Imhof SM, et al. Comparative genomic hybridisation divides retinoblastomas into a high and a low level chromosomal instability group. J Clin Pathol. 2003;56:26-30.

83. Zimonjic DB, Keck CL, Thorgeirsson SS, Popescu NC. Novel recurrent genetic imbalances in human hepatocellular carcinoma cell lines identified by comparative genomic hybridization. Hepatol. 1999;29:1208-14.

84. Poliseno L, Salmena L, Zhang J, Carver B, Haveman WJ, Pandolfi PP. A codingindependent function of gene and pseudogene mRNAs regulates tumour biology. Nature. 2010;465:1033-8.

85. Chen X, Guo X, Zhang H, Xiang Y, Chen J, Yin Y, et al. Role of miR-143 targeting KRAS in colorectal tumorigenesis. Oncogene. 2009;28:1385-92.

86. Long $X B$, Sun GB, Hu S, Liang GT, Wang N, Zhang XH, et al. Let-7a microRNA functions as a potential tumor suppressor in human laryngeal cancer. Oncol Rep. 2009;22:1189-95.

87. Booy EP, McRae EKS, Koul A, Lin F, McKenna SA. The long non-coding RNA BC200 (BCYRN1) is critical for cancer cell survival and proliferation. Mol Cancer. 2017;16:109.

88. $\mathrm{Yu} \mathrm{JH}$, Chen Y. Clinical significance of IncRNA BCYRN1 in colorectal cancer and its role in cell metastasis. Eur Rev Med Pharmacol Sci. 2019;23:9371-8.

89. Zhai $\mathrm{H}, \mathrm{Li}$ Y. BCYRN1 is correlated with progression and prognosis in gastric cancer. Biosci Rep. 2019;39:BSR20190505.

90. Gu L, Lu L, Zhou D, Liu Z. Long Noncoding RNA BCYRN1 promotes the proliferation of colorectal cancer cells via up-regulating NPR3 expression. Cell Physiol Biochem. 2018;48:2337-49.

91. Lang N, Wang C, Zhao J, Shi F, Wu T, Cao H. Long non-coding RNA BCYRN1 promotes glycolysis and tumor progression by regulating the miR-149/PKM2 axis in non-small-cell lung cancer. Mol Med Rep. 2020;21:1509-16.

92. Yang L, Zhang Y, Bao J, Feng J-F. Long non-coding RNA BCYRN1 exerts an oncogenic role in colorectal cancer by regulating the miR-204-3p/KRAS axis. Cancer Cell Int. 2020;20:453.

93. Li X, Deng S-J, Zhu S, Jin Y, Cui S-P, Chen J-Y, et al. Hypoxia-induced IncRNANUTF2P3-001 contributes to tumorigenesis of pancreatic cancer by derepressing the miR-3923/KRAS pathway. Oncotarget 2016;7:6000-14.

94. Rosenbluh J, Nijhawan D, Chen Z, Wong K-K, Masutomi K, Hahn WC. RMRP is a non-coding RNA essential for early murine development. PloS ONE. 2011;6: e26270.

95. Song H, Sun W, Ye G, Ding X, Liu Z, Zhang S, et al. Long non-coding RNA expression profile in human gastric cancer and its clinical significances. J Transl Med. 2013;11:225.

96. Shao $Y$, Ye M, Li Q, Sun W, Ye G, Zhang X, et al. LncRNA-RMRP promotes carcinogenesis by acting as a miR-206 sponge and is used as a novel biomarker for gastric cancer. Oncotarget. 2016;7:37812-24.

97. Wang $Y$, Luo $X$, Liu $Y$, Han G, Sun D. Long noncoding RNA RMRP promotes proliferation and invasion via targeting miR-1-3p in non-small-cell lung cancer. J Cell Biochem. 2019;120:15170-81.

98. Lin F, Yao L, Xiao J, Liu D, Ni Z. MiR-206 functions as a tumor suppressor and directly targets K-Ras in human oral squamous cell carcinoma. Onco Targets Ther. 2014;7:1583-91.

99. Meng Q, Ren M, Li Y, Song X. LncRNA-RMRP acts as an oncogene in lung cancer. PLoS One. 2016;11:e0164845

100. Lim YWS, Xiang X, Garg M, Le MT, Wong AL-A, Wang $L$, et al. The double-edged sword of H19 IncRNA: Insights into cancer therapy. Cancer Lett. 2020;500: 253-62.

101. Akao $Y$, Nakagawa $Y$, Naoe T. let-7 microRNA functions as a potential growth suppressor in human colon cancer cells. Biol Pharm Bull. 2006;29:903-6.

102. Luu C, Heinrich EL, Duldulao M, Arrington AK, Fakih M, Garcia-Aguilar J, et al. TP53 and let-7a micro-RNA regulate K-Ras activity in HCT116 colorectal cancer cells. PloS ONE. 2013;8:e70604-e.

103. Kallen AN, Zhou XB, Xu J, Qiao C, Ma J, Yan L, et al. The imprinted H19 IncRNA antagonizes let-7 microRNAs. Mol Cell. 2013;52:101-12.

104. Ma C, Nong K, Zhu H, Wang W, Huang X, Yuan Z, et al. H19 promotes pancreatic cancer metastasis by derepressing let-7's suppression on its target HMGA2mediated EMT. Tumour Biol: J Int Soc Oncodev Biol Med. 2014;35:9163-9.

105. Fish JE, Flores Suarez CP, Boudreau E, Herman AM, Gutierrez MC, Gustafson D, et al. Somatic gain of KRAS function in the endothelium is sufficient to cause vascular malformations that require MEK but Not PI3K signaling. Circ Res. 2020;127:727-43.

106. Shin KH, Bae SD, Hong HS, Kim RH, Kang MK, Park NH. miR-181a shows tumor suppressive effect against oral squamous cell carcinoma cells by downregulating K-ras. Biochemical biophysical Res Commun. 2011;404:896-902. 
107. Gastaldi C, Bertero T, Xu N, Bourget-Ponzio I, Lebrigand K, Fourre S, et al. miR193b/365a cluster controls progression of epidermal squamous cell carcinoma. Carcinogenesis. 2014;35:1110-20.

108. Ye Y, Guo J, Xiao P, Ning J, Zhang R, Liu P, et al. Macrophages-induced long noncoding RNA $\mathrm{H} 19$ up-regulation triggers and activates the miR-193b/MAPK1 axis and promotes cell aggressiveness in hepatocellular carcinoma. Cancer Lett. 2020;469:310-22.

109. Zhang W, Du M, Wang T, Chen W, Wu J, Li Q, et al. Long non-coding RNA LINC01133 mediates nasopharyngeal carcinoma tumorigenesis by binding to YBX1. Am J Cancer Res. 2019;9:779-90.

110. Zang C, Nie F-Q, Wang Q, Sun M, Li W, He J, et al. Long non-coding RNA LINC01133 represses KLF2, P21 and E-cadherin transcription through binding with EZH2, LSD1 in non small cell lung cancer. Oncotarget. 2016;7:11696-707.

111. Zhang J, Zhu N, Chen X. A novel long noncoding RNA LINC01133 is upregulated in lung squamous cell cancer and predicts survival. Tumour Biol: J Int Soc Oncodev Biol Med. 2015;36:7465-71.

112. Song Z, Zhang X, Lin Y, Wei Y, Liang S, Dong C. LINC01133 inhibits breast cancer invasion and metastasis by negatively regulating SOX4 expression through EZH2. J Cell Mol Med. 2019;23:7554-65.

113. Zhang JH, Li AY, Wei N. Downregulation of long non-coding RNA LINC01133 is predictive of poor prognosis in colorectal cancer patients. Eur Rev Med Pharmacol Sci. 2017;21:2103-7.

114. Zhang H, He Q-Y, Wang G-C, Tong D-K, Wang R-K, Ding W-B, et al. miR-422a inhibits osteosarcoma proliferation by targeting BCL2L2 and KRAS. Biosci Rep. 2018;38:BSR20170339.

115. Zeng HF, Qiu HY, Feng FB. Long noncoding RNA LINC01133 functions as an miR422a sponge to aggravate the tumorigenesis of human osteosarcoma. Oncol Res. 2018;26:335-43.

116. Wang Z, Jin J. LnCRNA SLCO4A1-AS1 promotes colorectal cancer cell proliferation by enhancing autophagy via miR-508-3p/PARD3 axis. Aging. 2019;11:4876-89.

117. Yu J, Han Z, Sun Z, Wang $Y$, Zheng M, Song C. LncRNA SLCO4A1-AS1 facilitates growth and metastasis of colorectal cancer through $\beta$-catenin-dependent Wnt pathway. J Exp Clin Cancer Res. 2018;37:222.

118. Tang R, Chen J, Tang M, Liao Z, Zhou L, Jiang J, et al. LncRNA SLCO4A1-AS1 predicts poor prognosis and promotes proliferation and metastasis via the EGFR/MAPK pathway in colorectal cancer. Int J Biol Sci. 2019;15:2885-96.

119. Fan $C$, Lin $Y$, Mao $Y$, Huang $Z$, Liu AY, Ma $H$, et al. MicroRNA-543 suppresses colorectal cancer growth and metastasis by targeting KRAS, MTA1 and HMGA2. Oncotarget. 2016;7:21825-39.

120. Graziano F, Canestrari E, Loupakis F, Ruzzo A, Galluccio N, Santini D, et al. Genetic modulation of the Let-7 microRNA binding to KRAS $3^{\prime}$-untranslated region and survival of metastatic colorectal cancer patients treated with salvage cetuximab-irinotecan. Pharmacogenomics J. 2010;10:458-64.

121. Zhang J, Huang J, Chen W, Hu Z, Wang X. miR-143-3p targets IncRNA PSMG3AS1 to inhibit the proliferation of hepatocellular carcinoma cells. Cancer Manag Res. 2020;12:6303-9.

122. Han P, Li J-W, Zhang B-M, Lv J-C, Li Y-M, Gu X-Y, et al. The IncRNA CRNDE promotes colorectal cancer cell proliferation and chemoresistance via miR$181 \mathrm{a}-5 \mathrm{p}$-mediated regulation of $\mathrm{Wnt} / \beta$-catenin signaling. Mol Cancer. 2017;16:9.

123. Wu J, Fantasia JE, Kaplan R. Oral manifestations of acute myelomonocytic leukemia: a case report and review of the classification of leukemias. J Periodontol. 2002;73:664-8.

124. Garg M, Nagata $Y$, Kanojia D, Mayakonda A, Yoshida K, Haridas Keloth S, et al. Profiling of somatic mutations in acute myeloid leukemia with FLT3-ITD at diagnosis and relapse. Blood. J Am Soc Hematol. 2015;126:2491-501.

125. Kirtonia A, Pandya G, Sethi G, Pandey AK, Das BC, Garg M. A comprehensive review of genetic alterations and molecular targeted therapies for the implementation of personalized medicine in acute myeloid leukemia. J Mol Med. 2020;98:1069-91.

126. Ward AF, Braun BS, Shannon KM. Targeting oncogenic Ras signaling in hematologic malignancies. Blood. 2012;120:3397-406.

127. Bhat AA, Younes SN, Raza SS, Zarif L, Nisar S, Ahmed I, et al. Role of non-coding RNA networks in leukemia progression, metastasis and drug resistance. Mol Cancer. 2020;19:1-21.

128. Hofmans M, Lammens T, Helsmoortel HH, Bresolin S, Cavé H, Flotho C, et al. The long non-coding RNA landscape in juvenile myelomonocytic leukemia. Haematologica. 2018;103:e501.

129. Mattias H, Lammens T, Depreter B, Wu Y, Erlacher M, Aurélie C, et al. Long noncoding RNAs as novel therapeutic targets in juvenile myelomonocytic leukemia. Sci Rep. 2021;11:2801

130. Kotzin JJ, Spencer SP, McCright SJ, Kumar DBU, Collet MA, Mowel WK, et al. The long non-coding RNA Morrbid regulates Bim and short-lived myeloid cell lifespan. Nature 2016;537:239-43.
131. Cai Z, Zhang C, Kotzin JJ, Williams A, Henao-Mejia J, Kapur R. Role of IncRNA Morrbid in PTPN11 (Shp2) E76K-driven juvenile myelomonocytic leukemia. Blood Adv. 2020;4:3246-51.

132. Wang H, Wang L, Zhang G, Lu C, Chu H, Yang R, et al. MALAT1/miR-101-3p/ MCL1 axis mediates cisplatin resistance in lung cancer. Oncotarget. 2018;9:7501.

133. Cruz-Miranda GM, Hidalgo-Miranda A, Bárcenas-López DA, Núñez-Enríquez JC, Ramírez-Bello J, Mejía-Aranguré JM, et al. Long non-coding RNA and acute leukemia. Int J Mol Sci. 2019;20:735.

134. Dieter C, Lourenco ED, Lemos NE. Association of long non-coding RNA and leukemia: a systematic review. Gene. 2020;735:144405.

135. Gao J, Wang F, Wu P, Chen Y, Jia Y. Aberrant LncRNA expression in leukemia. J Cancer. 2020;11:4284.

136. Pandya G, Kirtonia A, Sethi G, Pandey AK, Garg M. The implication of long noncoding RNAs in the diagnosis, pathogenesis and drug resistance of pancreatic ductal adenocarcinoma and their possible therapeutic potential. Biochim Biophys Acta Rev Cancer. 2020;1874:188423.

137. Siavoshi A, Taghizadeh M, Dookhe E, Piran M, Saliani M, Mohammad Ganji S. Network analysis of differential gene expression to identify hub genes in ovarian cancer. J Cell Mol Res. 2020;12:1-9.

138. Huang $R$, Zhou L, Chi Y, Wu H, Shi L. LncRNA profile study reveals a sevenIncRNA signature predicts the prognosis of patients with colorectal cancer. Biomark Res. 2020;8:1-16.

139. Leon LM, Gautier M, Allan R, llié M, Nottet N, Pons N, et al. Correction: The nuclear hypoxia-regulated NLUCAT1 long non-coding RNA contributes to an aggressive phenotype in lung adenocarcinoma through regulation of oxidative stress. Oncogene. 2019;38:7146--65.

140. Jiang $M-C$, Ni J-J, Cui W-Y, Wang B-Y, Zhuo W. Emerging roles of IncRNA in cancer and therapeutic opportunities. Am J Cancer Res. 2019;9:1354.

141. Jariwala N, Sarkar D. Emerging role of IncRNA in cancer: a potential avenue in molecular medicine. Ann Transl Med. 2016:4:286.

142. Bennett CF, Baker BF, Pham N, Swayze E, Geary RS. Pharmacology of antisense drugs. Annu Rev Pharmacol Toxicol. 2017;57:81-105.

143. Gilbert LA, Horlbeck MA, Adamson B, Villalta JE, Chen Y, Whitehead EH, et al. Genome-scale CRISPR-mediated control of gene repression and activation. Cell. 2014;159:647-61.

144. Elbashir SM, Harborth J, Lendeckel W, Yalcin A, Weber K, Tuschl T. Duplexes of 21-nucleotide RNAs mediate RNA interference in cultured mammalian cells. Nature. 2001;411:494-8.

145. Watanabe C, Cuellar TL, Haley B. Quantitative evaluation of first, second, and third generation hairpin systems reveals the limit of mammalian vector-based RNAi. RNA Biol. 2016;13:25-33.

146. Shi S, Li D, Li Y, Feng Z, Du Y, Nie Y. LncRNA CR749391 acts as a tumor suppressor to upregulate KLF6 expression via interacting with miR-181a in gastric cancer. Exp Therapeutic Med. 2020;19:569-78.

147. Zhou C, Wang X, Yang N, Xue S, Li W, Xie P. LncRNA LET function as a tumor suppressor in breast cancer development. Eur Rev Med Pharmacol Sci. 2018:22:6002-7.

148. Zhu S, Wang J, He Y, Meng N, Yan G-R. Peptides/proteins encoded by noncoding RNA: a novel resource bank for drug targets and biomarkers. Front Pharmacol. 2018;9:1295.

\section{ACKNOWLEDGEMENTS}

We are grateful to group members Niloufar Mosaddeghzedeh, Neda Sadat Kazemein Jasemi, Silke Pudewell, Mehrnaz Mehrabipour, and Farhad Bazgir from the Institute of Biochemistry and Molecular Biology II of the Heinrich Heine University for the critical reading of the manuscript.

\section{AUTHOR CONTRIBUTIONS}

M.S. and M.R.A. contributed to the conception, design of the article, and supervision, prepared the figures and the supplementary Table S1. A.M.B. contributed in paper writing and provided critical revision of the article. All authors gave final approval of the accepted version for publication.

\section{FUNDING}

This study was supported by the European Network on Noonan Syndrome and Related Disorders (NSEuroNet, grant number: 01GM1621B), and the German Federal Ministry of Education and Research (BMBF) - German Network of RASopathy Research (GeNeRARe, grant numbers: 01GM1902C). Open Access funding enabled and organized by Projekt DEAL. 


\section{COMPETING INTERESTS}

The authors declare no competing interests.

\section{ADDITIONAL INFORMATION}

Supplementary information The online version contains supplementary material available at https://doi.org/10.1038/s41417-021-00381-x.

Correspondence and requests for materials should be addressed to M.R.A.

Reprints and permission information is available at http://www.nature.com/ reprints

Publisher's note Springer Nature remains neutral with regard to jurisdictional claims in published maps and institutional affiliations.
Open Access This article is licensed under a Creative Commons Attribution 4.0 International License, which permits use, sharing, adaptation, distribution and reproduction in any medium or format, as long as you give appropriate credit to the original author(s) and the source, provide a link to the Creative Commons license, and indicate if changes were made. The images or other third party material in this article are included in the article's Creative Commons license, unless indicated otherwise in a credit line to the material. If material is not included in the article's Creative Commons license and your intended use is not permitted by statutory regulation or exceeds the permitted use, you will need to obtain permission directly from the copyright holder. To view a copy of this license, visit http://creativecommons. org/licenses/by/4.0/.

(c) The Author(s) 2021 